\title{
Global stability of May cooperative system with feedback controls
}

\author{
Rongyu Han ${ }^{1}$, Fengde Chen ${ }^{1 *}$, Xiangdong $\mathrm{Xie}^{2^{*}}$ and Zhanshuai Miao'
}

\section{"Correspondence:}

huanght0523@163.com;

ndsyxxd@163.com

${ }^{1}$ College of Mathematics and

Computer Science, Fuzhou

University, Fuzhou, Fujian 350116,

P.R. China

${ }^{2}$ Department of Mathematics,

Ningde Normal University, Ningde,

Fujian 352300, P.R. China

\begin{abstract}
In this paper, a May cooperative system with feedback controls is proposed and studied. The dynamic behaviors of the system are discussed by using the Lyapunov function method. If $b_{i} \neq 0, i=1,2$, we show that feedback control variables have no influence on the global stability of the unique positive equilibrium of the system, which means that feedback control variables only change the position of the positive equilibrium and retain its global stability property. If $b_{i}=0, i=1,2$, we can make the system which has a unique globally stable equilibrium or has unboundedly large solutions become globally stable. Some examples are given to illustrate the feasibility of the main results.
\end{abstract}

MSC: $34 \mathrm{C} 25 ; 92 \mathrm{D} 25 ; 34 \mathrm{D} 20 ; 34 \mathrm{D} 40$

Keywords: May cooperative system; feedback controls; Lyapunov function; global stability

\section{Introduction}

May [1] suggested the following set of equations to describe a pair of mutualists:

$$
\begin{aligned}
& \frac{d N_{1}}{d t}=r N_{1}\left[1-\frac{N_{1}}{K_{1}+\alpha N_{2}}\right], \\
& \frac{d N_{2}}{d t}=r N_{2}\left[1-\frac{N_{2}}{K_{2}+\beta N_{1}}\right],
\end{aligned}
$$

where $N_{1}, N_{2}$ are the densities of the species, respectively. $r, K_{i}, \alpha, \beta, i=1,2$, are positive constants. He showed that if the coefficients of system (1.1) satisfy

$$
\alpha \beta<1,
$$

then system (1.1) has a global stability equilibrium point $\left(N_{1}^{*}, N_{2}^{*}\right)$, where

$$
N_{1}^{*}=\frac{K_{1}+\alpha K_{2}}{1-\alpha \beta}, \quad N_{2}^{*}=\frac{K_{2}+\beta K_{1}}{1-\alpha \beta} .
$$

If the amount of mutualistic interaction satisfies

$$
\alpha \beta \geq 1,
$$

(c) 2015 Han et al. This article is distributed under the terms of the Creative Commons Attribution 4.0 International License (http://creativecommons.org/licenses/by/4.0/), which permits unrestricted use, distribution, and reproduction in any medium, provided you give appropriate credit to the original author(s) and the source, provide a link to the Creative Commons license, and indicate if changes were made. 
then system (1.1) will 'run away', with both populations growing unboundedly large. So May brought the density restriction to the system and proposed the following system [2]:

$$
\begin{aligned}
& \dot{x}=r_{1} x\left[1-\frac{x}{K_{1}+\alpha_{1} y}-\varepsilon_{1} x\right], \\
& \dot{y}=r_{2} y\left[1-\frac{y}{K_{2}+\alpha_{2} x}-\varepsilon_{2} y\right],
\end{aligned}
$$

where $r_{i}, K_{i}, \alpha_{i}, \varepsilon_{i}, i=1,2$ are positive constants. He showed that system (1.4) has a global stability equilibrium point.

Since that many excellent results concerned with the dynamic behaviors of May cooperative system are obtained. Cui and Chen [3] addressed the nonautonomous system

$$
\begin{aligned}
& \dot{u}=r_{1}(t) u\left[1-\frac{u}{a_{1}(t)+b_{1}(t) v}-c_{1}(t) u\right], \\
& \dot{v}=r_{2}(t) v\left[1-\frac{v}{a_{2}(t)+b_{2}(t) u}-c_{2}(t) v\right],
\end{aligned}
$$

under the assumption that $r_{i}(t), a_{i}(t), b_{i}(t), c_{i}(t), i=1,2$, are all continuous $T$-periodic functions, and they obtained sufficient conditions which guarantee the existence of a unique globally asymptotically stable strictly positive periodic solution. In [4], Cui further incorporated continuous time delays and generalized it to $n$ species. In $[5,6]$, the authors incorporated discrete time delays in interspecies interaction and investigated the positive periodic and positive almost periodic solution of system.

On the other hand, in the real world, ecosystems are continuously disturbed by the unpredictable force. It is mostly humans' interference. Furthermore human production activities is an important reason of species extinction $[7,8]$. In some situations, one may wish to retain its stability. This is the significance in the control procedure of ecology balance. Therefore, scholars introduced the feedback control variables to the system. The dynamic behaviors of the Lotka-Volterra cooperative system with feedback controls have been discussed extensively; see [9-13]. In [10], Chen et al. first proposed the nonautonomous $n$-species cooperation system with continuous delays and feedback controls as follows:

$$
\begin{aligned}
\frac{d x_{i}(t)}{d t}= & r_{i}(t) x_{i}(t)\left[1-\frac{x_{i}(t)}{a_{i}(t)+\sum_{j=1, j \neq i}^{n} b_{i j} \int_{-T_{i j}}^{0} K_{i j}(s) x_{j}(t+s) d s}-c_{i}(t) x_{i}(t)\right] \\
& -d_{i}(t) u_{i}(t) x_{i}(t)-e_{i}(t) x_{i}(t) \int_{-\tau_{i}}^{0} H_{i}(s) u_{i}(t+s) d s, \\
\frac{d u_{i}(t)}{d t}= & -\alpha_{i}(t) u_{i}(t)+\beta_{i}(t) x_{i}(t)+\gamma_{i}(t) \int_{-\eta_{i}}^{0} G_{i}(s) x_{i}(t+s) d s,
\end{aligned}
$$

where $x_{i}(t), i=1, \ldots, n$ are the densities of the cooperation species $X_{i} . u_{i}(t), i=1, \ldots, n$ are the feedback control variables. They obtained a set of complicated sufficient conditions to guarantee the permanence and global attractivity of the system. Recently, by applying a new integral inequality, Chen and Xie [14] showed that in the above system, the feedback control variables have no influence on the persistent property of system (1.6). With regard to an autonomous system, since 2003, Gopalsamy and Weng [15] introduced feedback 
control variables into a two species competition system as follows:

$$
\begin{aligned}
& \frac{d x_{1}(t)}{d t}=x_{1}(t)\left(b_{1}-a_{11} x_{1}(t)-a_{12} x_{2}(t)-\alpha_{1} u_{1}(t)\right), \\
& \frac{d x_{2}(t)}{d t}=x_{2}(t)\left(b_{2}-a_{21} x_{1}(t)-a_{22} x_{2}(t)-\alpha_{2} u_{2}(t)\right), \\
& \frac{d u_{1}(t)}{d t}=-\eta_{1} u_{1}(t)+a_{1} x_{1}(t), \\
& \frac{d u_{2}(t)}{d t}=-\eta_{2} u_{2}(t)+a_{2} x_{2}(t) .
\end{aligned}
$$

They obtained sufficient conditions to guarantee the existence of a globally attracting positive equilibrium of the system with feedback controls. Chen and Chen [16] discussed the global stability of a unique interior equilibrium for a Leslie-Gower predator-prey model with feedback controls:

$$
\begin{aligned}
& \dot{N}_{1}(t)=\left(r_{1}-a_{1} N_{2}-b_{1} N_{1}-c_{1} u_{1}\right) N_{1}, \\
& \dot{N}_{2}(t)=\left(r_{2}-a_{2} \frac{N_{2}}{N_{1}}-c_{2} u_{2}\right) N_{2}, \\
& \dot{u}_{1}(t)=-f_{1} u_{1}+g_{1} N_{1}, \\
& \dot{u}_{2}(t)=-f_{2} u_{2}+g_{2} N_{2} .
\end{aligned}
$$

They showed that under the assumption $a_{2} b_{1} \geq a_{1} r_{2}$, the unique interior equilibrium of system (1.8) is globally stable. However, the authors invoked numerical calculations to found that the unique interior equilibrium of system (1.8) is globally stable also under the condition that $a_{2} b_{1}<a_{1} r_{2}$ holds. So the condition $a_{2} b_{1} \geq a_{1} r_{2}$ is unnecessary for the globally stable property of system (1.8). This is a question to be studied in the future. Recently, Li et al. [8] proposed and studied the following competitive system with feedback controls:

$$
\begin{aligned}
& \dot{x}_{1}(t)=x_{1}(t)\left(b_{1}-a_{11} x_{1}(t)-a_{12} \int_{0}^{+\infty} K_{1}(s) x_{2}(t-s) d s-c_{1} u_{1}(t)\right), \\
& \dot{x}_{2}(t)=x_{2}(t)\left(b_{2}-a_{21} \int_{0}^{+\infty} K_{2}(s) x_{1}(t-s) d s-a_{22} x_{2}(t)-c_{2} u_{2}(t)\right), \\
& \dot{u}_{1}(t)=-e_{1} u_{1}(t)+d_{1} x_{1}(t) \\
& \dot{u}_{2}(t)=-e_{2} u_{2}(t)+d_{2} x_{2}(t)
\end{aligned}
$$

where $b_{i}, a_{i j}, c_{i}, e_{i}, d_{i}, i, j=1,2$ are positive constants. $x_{i}(t), i=1,2$, denote the densities of the populations $x_{i}(t) . u_{i}(t), i=1,2$, denote feedback control variables. They showed that if the Lotka-Volterra competitive system is globally stable, the feedback control variables had no influence on the global stability of the system (1.9). If the Lotka-Volterra competitive system is showing extinction, they can make the extinct species become globally stable or still keep the property of extinction. They showed that the feedback control variables play an important role on the dynamic behavior of the system (1.9).

It brings to our attention that in systems (1.4) and (1.1), we may ask: how do feedback control variables affect the global stability of the May cooperative system? To find an answer to this question, we consider a May cooperative system with feedback controls as 
follows:

$$
\begin{aligned}
& \frac{d x_{1}}{d t}=r_{1} x_{1}\left[1-b_{1} x_{1}-\frac{x_{1}}{\alpha x_{2}+k_{1}}-c_{1} u_{1}\right], \\
& \frac{d x_{2}}{d t}=r_{2} x_{2}\left[1-b_{2} x_{2}-\frac{x_{2}}{\beta x_{1}+k_{2}}-c_{2} u_{2}\right], \\
& \frac{d u_{1}}{d t}=-\eta_{1} u_{1}+e_{1} x_{1}, \\
& \frac{d u_{2}}{d t}=-\eta_{2} u_{2}+e_{2} x_{2},
\end{aligned}
$$

where $r_{i}, b_{i}, \alpha, \beta, k_{i}, c_{i}, e_{i}, \eta_{i}, i=1,2$, are positive constants. $x_{i}(t), i=1,2$, are the densities of the species at time $t . u_{i}(t), i=1,2$, denote feedback control variables.

System (1.10) satisfies the initial values

$$
x_{i}(0)>0, \quad u_{i}(0)>0, \quad i=1,2 .
$$

Obviously, the solutions of system (1.10) with initial values (1.11) are positive for all $t \geq 0$.

The rest of the paper is organized as follows. We will state and prove the main results in next section. In Section 3, numerical simulations are presented to illustrate our results. We end this work by a brief conclusion.

\section{Main results}

Lemma 2.1 System (1.10) admits a unique positive equilibrium $P\left(x_{1}^{*}, x_{2}^{*}, u_{1}^{*}, u_{2}^{*}\right)$.

Proof Obviously, $P\left(x_{1}^{*}, x_{2}^{*}, u_{1}^{*}, u_{2}^{*}\right)$ satisfies the following equations:

$$
\left\{\begin{array}{l}
1-b_{1} x_{1}-\frac{x_{1}}{\alpha x_{2}+k_{1}}-c_{1} u_{1}=0 \\
1-b_{2} x_{2}-\frac{x_{2}}{\beta x_{1}+k_{2}}-c_{2} u_{2}=0 \\
-\eta_{1} u_{1}+e_{1} x_{1}=0 \\
-\eta_{2} u_{2}+e_{2} x_{2}=0
\end{array}\right.
$$

By the third and the fourth equations of (2.1), we have $u_{i}=\frac{e_{i} x_{i}}{\eta_{i}}, i=1,2$. Substituting them into the first and the second equations of (2.1), respectively, we have

$$
x_{2}=\frac{k_{2}+\beta x_{1}}{1+A_{2} k_{2}+A_{2} \beta x_{1}}
$$

and

$$
\left(1-A_{1} x_{1}\right)\left(k_{1}+\alpha x_{2}\right)=x_{1},
$$

where $A_{i}=b_{i}+\frac{c_{i} e_{i}}{\eta_{i}}, i=1,2$. Substituting (2.2) into (2.3), we have

$$
D x_{1}^{2}+E x_{1}+F=0 \text {, }
$$

where

$$
\begin{aligned}
& D=\beta\left(A_{2}+k_{1} A_{1} A_{2}+A_{1} \alpha\right), \quad F=-\left(k_{1}+k_{1} k_{2} A_{2}+k_{2} \alpha\right), \\
& E=k_{1}\left(A_{1}-A_{2} \beta\right)+k_{2}\left(A_{2}+A_{1} \alpha+k_{1} A_{1} A_{2}\right)+(1-\alpha \beta) .
\end{aligned}
$$


From $D>0, F<0$, we see that (2.4) admits a unique positive solution $x_{1}^{*}$. Substituting $x_{1}^{*}$ to (2.2), we have a unique positive solution $x_{2}^{*}$. So system (1.10) admits a unique positive equilibrium $P\left(x_{1}^{*}, x_{2}^{*}, u_{1}^{*}, u_{2}^{*}\right)$, where $u_{i}^{*}=\frac{e_{i} x_{i}^{*}}{\eta_{i}}, i=1,2$, which completes the proof.

Before we state and prove the global stability of this work, we need to state a definition and a useful lemma.

Definition 2.2 [16] A matrix $A=\left(\left(a_{i j}\right)_{n \times n}\right)$ is said to be an $M$ matrix if $a_{i j} \leq 0, i \neq j, i, j=$ $1,2, \ldots, n$, and any one of the following conditions holds:

(1) all of the eigenvalues of the matrix $A$ have positive real parts;

(2) the order principal minor of matrix $A$ is positive;

(3) matrix $A$ is nonsingular and $A^{-1} \geq 0$;

(4) there exists a vector $x>0$ such that $A x>0$;

(5) there exists a vector $y>0$ such that $A^{T} y>0$.

Lemma 2.3 [17] If $A$ is an $M$ matrix, then there exists a positive diagonal matrix $D=$ $\operatorname{diag}\left(d_{1}, d_{2}, \ldots, d_{n}\right), d_{i}>0, i=1, \ldots, n$, such that matrix $B=\frac{1}{2}\left(D A+A^{T} D\right)$ is positive definite.

Theorem 2.4 The unique positive equilibrium $P\left(x_{1}^{*}, x_{2}^{*}, u_{1}^{*}, u_{2}^{*}\right)$ of system (1.10) is globally stable.

Proof Now let us construct a Lyapunov function

$$
\begin{aligned}
V(t)= & \beta_{1} \int_{x_{1}^{*}}^{x_{1}} \frac{\theta-x_{1}^{*}}{\theta} d \theta+\beta_{2} \int_{x_{2}^{*}}^{x_{2}} \frac{\theta-x_{2}^{*}}{\theta} d \theta+\beta_{3} \int_{u_{1}^{*}}^{u_{1}}\left(\theta-u_{1}^{*}\right) d \theta \\
& +\beta_{4} \int_{u_{2}^{*}}^{u_{2}}\left(\theta-u_{2}^{*}\right) d \theta,
\end{aligned}
$$

where $\beta_{i}, i=1, \ldots, 4$ are positively undetermined coefficients. Calculating the upper right derivative of $V(t)$ along the solution of system (1.10), we have

$$
\begin{aligned}
D^{+} V(t)= & \beta_{1} r_{1}\left(x_{1}-x_{1}^{*}\right)\left[-b_{1}\left(x_{1}-x_{1}^{*}\right)-\frac{x_{1}}{\alpha x_{2}+k_{1}}+\frac{x_{1}^{*}}{\alpha x_{2}^{*}+k_{1}}-c_{1}\left(u_{1}-u_{1}^{*}\right)\right] \\
& +\beta_{2} r_{2}\left(x_{2}-x_{2}^{*}\right)\left[-b_{2}\left(x_{2}-x_{2}^{*}\right)-\frac{x_{2}}{\beta x_{1}+k_{2}}+\frac{x_{2}^{*}}{\beta x_{1}^{*}+k_{2}}-c_{2}\left(u_{2}-u_{2}^{*}\right)\right] \\
& +\beta_{3}\left(u_{1}-u_{1}^{*}\right)\left[-\eta_{1}\left(u_{1}-u_{1}^{*}\right)+e_{1}\left(x_{1}-x_{1}^{*}\right)\right] \\
& +\beta_{4}\left(u_{2}-u_{2}^{*}\right)\left[-\eta_{2}\left(u_{2}-u_{2}^{*}\right)+e_{2}\left(x_{2}-x_{2}^{*}\right)\right]
\end{aligned}
$$

we take $\beta_{i+2}=\frac{\beta_{i} r_{i} c_{i}}{e_{i}}, i=1,2$, then

$$
\begin{aligned}
D^{+} V(t)= & -\beta_{1} r_{1}\left[b_{1}+\frac{1}{\alpha x_{2}+k_{1}}\right]\left(x_{1}-x_{1}^{*}\right)^{2}-\beta_{2} r_{2}\left[b_{2}+\frac{1}{\beta x_{1}+k_{2}}\right] \\
& \times\left(x_{2}-x_{2}^{*}\right)^{2}+\left[\frac{\beta_{1} r_{1} \alpha x_{1}^{*}}{\left(\alpha x_{2}^{*}+k_{1}\right)\left(\alpha x_{2}+k_{1}\right)}+\frac{\beta_{2} r_{2} \beta x_{2}^{*}}{\left(\beta x_{1}^{*}+k_{2}\right)\left(\beta x_{1}+k_{2}\right)}\right] \\
& \times\left(x_{1}-x_{1}^{*}\right)\left(x_{2}-x_{2}^{*}\right)-\beta_{3} \eta_{1}\left(u_{1}-u_{1}^{*}\right)^{2}-\beta_{4} \eta_{2}\left(u_{2}-u_{2}^{*}\right)^{2} \\
\leq & -\frac{1}{2} Y^{T}\left(D G+G^{T} D\right) Y-\beta_{3} \eta_{1}\left(u_{1}-u_{1}^{*}\right)^{2}-\beta_{4} \eta_{2}\left(u_{2}-u_{2}^{*}\right)^{2},
\end{aligned}
$$


where $Y=\left(\left|x_{1}-x_{1}^{*}\right|,\left|x_{2}-x_{2}^{*}\right|\right)^{T}, D=\operatorname{diag}\left(\beta_{1}, \beta_{2}\right), \beta_{i}>0, i=1,2$, and

$$
G=\left(\begin{array}{ll}
a_{11} & a_{12} \\
a_{21} & a_{22}
\end{array}\right),
$$

where

$$
\begin{aligned}
& a_{11}=r_{1}\left(b_{1}+\frac{1}{\alpha x_{2}+k_{1}}\right), \\
& a_{12}=-\frac{r_{1} \alpha x_{1}^{*}}{\left(\alpha x_{2}^{*}+k_{1}\right)\left(\alpha x_{2}+k_{1}\right)}, \\
& a_{21}=-\frac{r_{2} \beta x_{2}^{*}}{\left(\beta x_{1}^{*}+k_{2}\right)\left(\beta x_{1}+k_{2}\right)}, \\
& a_{22}=r_{2}\left(b_{2}+\frac{1}{\beta x_{1}+k_{2}}\right) .
\end{aligned}
$$

Note first that the off-diagonal elements of the matrix $G$ are $a_{21}$ and $a_{21}$, which are negative. Furthermore, by simple algebraic computation, the two order principal minors of the matrix $G$ are

$$
\begin{aligned}
& a_{11}=r_{1}\left(b_{1}+\frac{1}{\alpha x_{2}+k_{1}}\right)>0, \\
& \left|\begin{array}{ll}
a_{11} & a_{12} \\
a_{21} & a_{22}
\end{array}\right|=r_{1} r_{2} b_{1} b_{2}+\frac{r_{1} r_{2} b_{1}}{\beta x_{1}+k_{2}}+\frac{r_{1} r_{2} b_{2}}{\alpha x_{2}+k_{1}}+\frac{r_{1} r_{2}}{\left(\beta x_{1}+k_{2}\right)\left(\alpha x_{2}+k_{1}\right)} \\
& -\frac{r_{1} r_{2} \beta x_{1}^{*} \alpha x_{2}^{*}}{\left(\beta x_{1}+k_{2}\right)\left(\alpha x_{2}+k_{1}\right)\left(\beta x_{1}^{*}+k_{2}\right)\left(\alpha x_{2}^{*}+k_{1}\right)}>0 \text {. }
\end{aligned}
$$

From Definition 2.2, it follows that $G$ is an M matrix; according to Lemma 2.3, there exists a positive diagonal matrix $D=\operatorname{diag}\left(\beta_{1}, \beta_{2}\right), \beta_{i}>0, i=1,2$, such that the matrix $\frac{1}{2}\left(D G+G^{T} D\right)$ is positive definite. So $\frac{d V}{d t}<0$ strictly for all $x_{1}>0, x_{2}>0, u_{1}>0, u_{2}>0$, except the positive equilibrium $P\left(x_{1}^{*}, x_{2}^{*}, u_{1}^{*}, u_{2}^{*}\right)$, where $\frac{d V}{d t}=0$. So $V(t)$ satisfies Lyapunov's asymptotic stability theorem [18] and the unique interior equilibrium $P\left(x_{1}^{*}, x_{2}^{*}, u_{1}^{*}, u_{2}^{*}\right)$ is globally stable, which completes the proof.

Next we consider the dynamic behaviors of system (1.1) incorporate feedback control variables, in system (1.10), set $b_{i}=0, i=1,2$, and we have

$$
\begin{aligned}
& \frac{d x_{1}}{d t}=r_{1} x_{1}\left[1-\frac{x_{1}}{\alpha x_{2}+k_{1}}-c_{1} u_{1}\right] \\
& \frac{d x_{2}}{d t}=r_{2} x_{2}\left[1-\frac{x_{2}}{\beta x_{1}+k_{2}}-c_{2} u_{2}\right] \\
& \frac{d u_{1}}{d t}=-\eta_{1} u_{1}+e_{1} x_{1}, \\
& \frac{d u_{2}}{d t}=-\eta_{2} u_{2}+e_{2} x_{2} .
\end{aligned}
$$

Lemma 2.5 System (2.6) admits a unique equilibrium $P_{1}\left(x_{10}, x_{20}, u_{10}, u_{20}\right)$. 
Proof Obviously, $P_{1}\left(x_{10}, x_{20}, u_{10}, u_{20}\right)$ satisfies the following equations:

$$
\left\{\begin{array}{l}
1-\frac{x_{1}}{\alpha x_{2}+k_{1}}-c_{1} u_{1}=0 \\
1-\frac{x_{2}}{\beta x_{1}+k_{2}}-c_{2} u_{2}=0 \\
-\eta_{1} u_{1}+e_{1} x_{1}=0 \\
-\eta_{2} u_{2}+e_{2} x_{2}=0
\end{array}\right.
$$

After simple algebraic computations, we have

$$
D_{1} x_{1}^{2}+E_{1} x_{1}+F_{1}=0
$$

where

$$
\begin{aligned}
& D_{1}=\beta\left(B_{2}+k_{1} B_{1} B_{2}+B_{1} \alpha\right), \quad F_{1}=-\left(k_{1}+k_{1} k_{2} B_{2}+k_{2} \alpha\right), \\
& E_{1}=k_{1}\left(B_{1}-B_{2} \beta\right)+k_{2}\left(B_{2}+B_{1} \alpha+k_{1} B_{1} B_{2}\right)+(1-\alpha \beta),
\end{aligned}
$$

and $B_{i}=\frac{c_{i} e_{i}}{\eta_{i}}, i=1,2$. From $D_{1}>0, F_{1}<0$, we see that (2.8) admits a unique positive solution $x_{10}$. Similar to the analysis of Lemma 2.1, system (2.6) admits a unique positive equilibrium $P_{1}\left(x_{10}, x_{20}, u_{10}, u_{20}\right)$.

Theorem 2.6 The unique positive equilibrium $P_{1}\left(x_{10}, x_{20}, u_{10}, u_{20}\right)$ of system (2.6) is globally stable.

Proof The proof of Theorem 2.6 is similar to that of Theorem 2.4, and we omit the details here.

\section{Examples}

The following three examples show the feasibility of our main results.

Example 3.1 Consider the following equations:

$$
\dot{x_{1}}=x_{1}\left(1-2 x_{1}-\frac{2 x_{1}}{x_{2}+4}\right), \quad \dot{x_{2}}=x_{2}\left(2-x_{2}-\frac{3 x_{2}}{x_{1}+1}\right) \text {. }
$$

By calculation, there exists a unique positive equilibrium of system (3.1), which is globally stable. Figure 1 shows the dynamic behavior of system (3.1).

Figure 1 Dynamic behavior of the solution $\left(x_{1}(t), x_{2}(t)\right)$ to system (3.1) with the initial conditions $\left(x_{1}(0), x_{2}(0)\right)=(0.5,0.2),(0.3$, $0.4)$ and $(0.6,0.8)$, respectively.

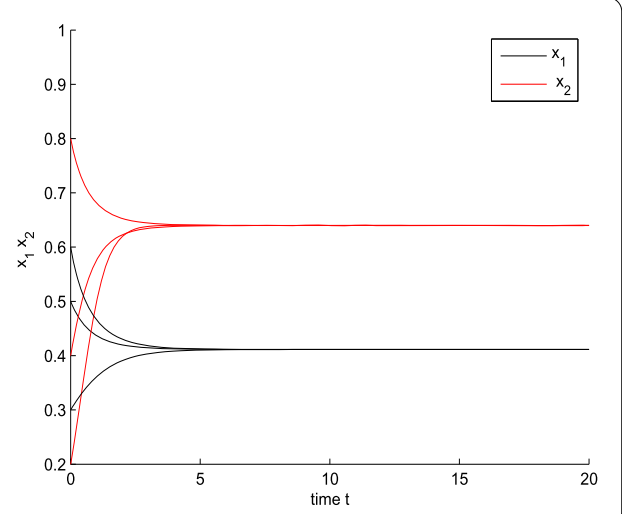




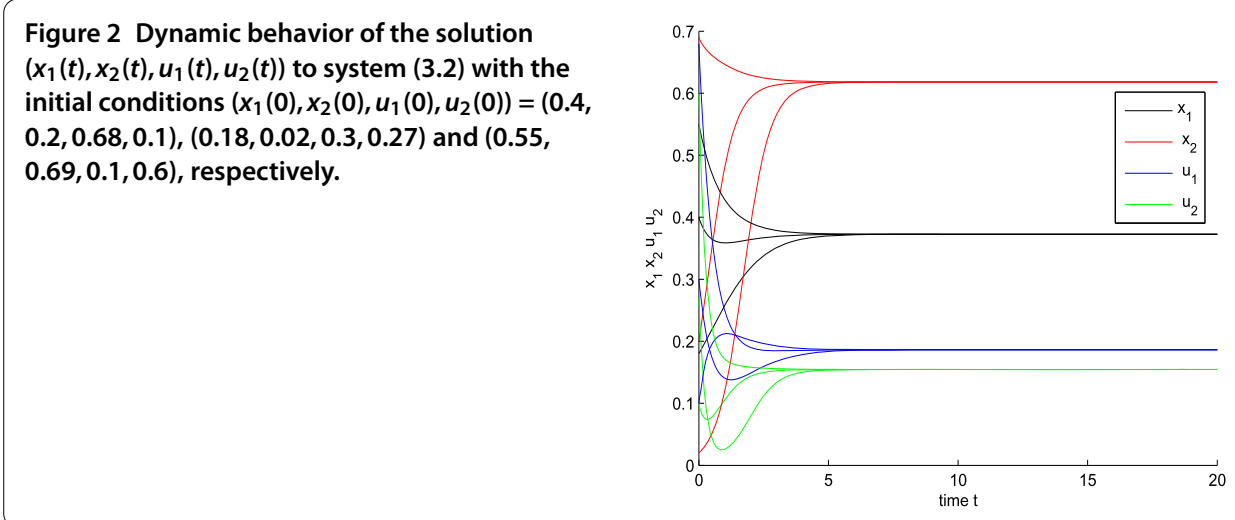

Example 3.2 Now let us further incorporate the feedback control variables to system (3.1) and consider the following system:

$$
\begin{aligned}
& \dot{x_{1}}=x_{1}\left(1-2 x_{1}-\frac{2 x_{1}}{x_{2}+4}-0.5 u_{1}\right), \\
& \dot{x_{2}}=x_{2}\left(2-x_{2}-\frac{3 x_{2}}{x_{1}+1}-0.2 u_{2}\right), \\
& \dot{u}_{1}=-2 u_{1}+x_{1}, \\
& \dot{u}_{2}=-4 u_{2}+x_{2} .
\end{aligned}
$$

From Theorem 2.4, the positive equilibrium of system (3.2) is globally stable. Figure 2 shows the dynamic behavior of system (3.2).

Example 3.3 Consider the following equations:

$$
\begin{aligned}
& \dot{x_{1}}=x_{1}\left(2-\frac{x_{1}}{x_{2}+2}-0.2 u_{1}\right), \\
& \dot{x_{2}}=x_{2}\left(3-\frac{2 x_{2}}{x_{1}+1}-0.5 u_{2}\right), \\
& \dot{u_{1}}=-3 u_{1}+2 x_{1}, \\
& \dot{u_{2}}=-2 u_{2}+x_{2} .
\end{aligned}
$$

From Theorem 2.6, there exists a unique positive equilibrium of system (3.3), which is globally stable. Figure 3 shows the dynamic behavior of system (3.3).

\section{Conclusion}

In this paper, we propose and study May cooperative system with feedback controls. In Theorem 2.4, by constructing a suitable Lyapunov function, we show that feedback control variables have no influence on the global stability of the system. Our result improve the corresponding result of Chen et al. [10]. In [16], Chen and Chen have a conjecture that the condition $a_{2} b_{1} \geq a_{1} r_{2}$ is not needed to ensure the global stability of the unique interior equilibrium. In this paper, corresponding to a May cooperative system, we give a strict proof of an affirmative answer which is without any conditions. Compared with Chen et 
Figure 3 Dynamic behavior of the solution $\left(x_{1}(t), x_{2}(t), u_{1}(t), u_{2}(t)\right)$ to system (3.3) with the initial conditions $\left(x_{1}(0), x_{2}(0), u_{1}(0), u_{2}(0)\right)=(2,1$, $4,6),(6.4,5.2,7,0.4)$ and $(9.2,4.1,0.9,3.3)$, respectively.

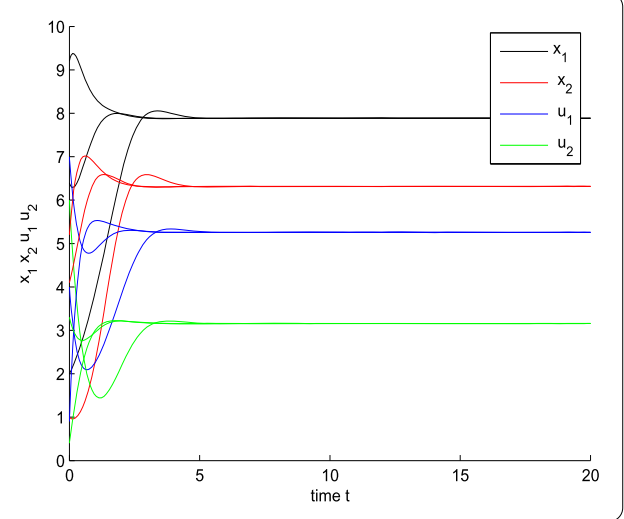

al. $[13,14]$, the authors showed that the feedback control variables have no influence on the permanence of the cooperation system. We have a further insight.

System (3.3) shows that

$$
1 \leq \alpha \beta=3,
$$

which implies condition (1.3) holds, that is, both populations are growing unboundedly large. However, Figure 3 and Theorem 2.6 show that the unique positive equilibrium is globally stable, which implies the feedback control variables which make both populations growing unboundedly large now become globally stable.

\section{Competing interests}

The authors declare that there is no conflict of interests.

\section{Authors' contributions}

All authors contributed equally to the writing of this paper. All authors read and approved the final manuscript.

\section{Acknowledgements}

The authors are grateful to anonymous referees for their excellent suggestions, which greatly improve the presentation of the paper. Also, the research was supported by the Natural Science Foundation of Fujian Province (2015J010121, 2015J01019).

Received: 12 June 2015 Accepted: 30 September 2015 Published online: 24 November 2015

\section{References}

1. May, RM: Theoretical Ecology: Principles and Applications. Sounders, Philadelphia (1976)

2. Chen, LS, Song, XY, Lu, ZY: Mathematical Models and Methods in Ecology. Sichuan Science and Technology Press, Sichuan (2003) (in Chinese)

3. Cui, JA, Chen, LS: Global asymptotic stability in a nonautonomous cooperative system. Sys. Sci. Math. Sci. 6(1), 44-51 (1993)

4. Cui, JA: Global asymptotic stability in $n$-species cooperative system with time delays. Sys. Sci. Math. Sci. 7(1), 45-48 (1994)

5. Wei, FY, Wang, K: Asymptotically periodic solution of N-species cooperation system with time delay. Nonlinear Anal., Real World Appl. 7(4), 591-596 (2006)

6. Chen, HB, He, WS, Yang, LX, Liu, XJ: Asymptotically almost periodic solution of $N$ population reciprocity system. J. Tianshui Norm. Univ. 29(2), 24-27 (2009)

7. Zhao, L, Xie, XX, Yang, LY, Chen, FD: Dynamic behaviors of a discrete Lotka-Volterra competition system with infinite delays and single feedback control. Abstr. Appl. Anal. 2014, Article ID 867313 (2014)

8. Li, Z, Han, MA, Chen, FD: Influence of feedback control on an autonomous Lotka-Volterra competitive system with infinite delays. Nonlinear Anal., Real World Appl. 14(1), 402-413 (2013)

9. Chen, FD: Permanence of a discrete $N$-species cooperation system with time delays and feedback controls. Appl. Math. Comput. 186(1), 23-29 (2007)

10. Chen, FD, Liao, XY, Huang, ZK: The dynamic behavior of $N$-species cooperation system with continuous time delays and feedback controls. Appl. Math. Comput. 181(2), 803-815 (2006)

11. Chen, FD, Yang, JH, Chen, LJ: On a mutualism model with feedback controls. Appl. Math. Comput. 214(2), 581-587 (2009) 
12. Chen, FD, Xie, XD: Study on the Dynamic Behaviors of Cooperation Population Modeling. Science Press, Beijing (2014) (in Chinese)

13. Chen, $L$, Xie, XD, Chen, $\mathrm{L}$ : Feedback control variables have no influence on the permanence of a discrete $\mathrm{N}$-species cooperation system. Discrete Dyn. Nat. Soc. 2009, Article ID 306425 (2009)

14. Chen, $L$, Xie, XD: Permanence of a $n$-species cooperation system with continuous time delays and feedback controls. Nonlinear Anal., Real World Appl. 12(1), 34-38 (2011)

15. Gopalsamy, K, Weng, PX: Global attractivity in a competition system with feedback controls. Comput. Math. Appl. 45(4-5), 665-676 (2003)

16. Chen, LJ, Chen, FD: Global stability of a Leslie-Gower predator-prey model with feedback controls. Appl. Math. Lett. 22(9), 1330-1334 (2009)

17. Chen, LS, Song, XY, Lu, ZY: Mathematical Models and Methods in Ecology. Sichuan Science and Technology Press, Sichuan (2003) (in Chinese)

18. Liao, XX: Theory Methods and Application of Stability. Huazhong University of Science and Technology Press, Wuhan (2004) (in Chinese)

Submit your manuscript to a SpringerOpen ${ }^{\odot}$ journal and benefit from:

- Convenient online submission

- Rigorous peer review

- Immediate publication on acceptance

- Open access: articles freely available online

- High visibility within the field

- Retaining the copyright to your article 